\title{
QoS-RRC: Integrated QoS Routing and Resource Provisioning Mechanism for Future Internet
}

\author{
Augusto Neto \\ Instituto de Informática - UFG \\ Goiânia, Brasil \\ augusto@inf.ufg.br \\ Sérgio Figueiredo \\ Instituto de Telecomunicações \\ Aveiro, Portugal \\ sfigueiredo@av.it.pt
}

\author{
Leandro Maçal \\ Instituto de Informática - UFG \\ Goiânia, Brasil \\ leandro@inf.ufg.br \\ Rui L. Aguiar \\ Universidade de Aveiro \\ Aveiro, Portugal \\ ruilaa@det.ua.pt
}

\begin{abstract}
Generic Path (GP) is a new connectivity paradigm proposed to facilitate the inclusion of new applications and services abstracting communications between entities, regardless of their location or architectural layer. This paper enhances the GP architecture with a new mechanism integrating QoS-Routing and Resource Control (QoS-RRC) strategies to dynamically control GP session requirements regarding QoS and connectivity. This solution uses overprovisioning and admission control, and is aimed at multiparty time-sensitive sessions. Initial performance evaluation was carried out in Network Simulator v.2 (NS-2), showing capabilities in reducing overall signaling load in comparison with a value-added per-flow approach.
\end{abstract}

Keywords- Future Internet, QoS Routing, Resource Allocation, Multi-party

\section{INTRODUCTION}

The growth in the number of Internet services and applications accessed ubiquitously (at anytime, anywhere and with any end device) has raised key performance issues related to the difficulty of having uninterruptible and reliable connections. Besides, the time-sensitiveness characteristics of multimedia are hardly supported by current Internet model. Resource-constrained sessions require bandwidth guarantees and robustness for data propagation beyond the traditional best-effort transport paradigm. Thus, Quality of Service (QoS) mechanisms are of paramount importance, and network resources must be provisioned so that sessions can match service level agreements (SLAs). Moreover, the bandwidth-optimized features of multicast, where multiple users can receive the same content simultaneously without affecting the network performance, makes it an interesting solution, deploying packet duplications only in strategic nodes.

Attempts to overcome or alleviate the above shortcomings pushed the deployment of some functions (QoS, multicast), not natively supported by Internet's conversational model, on top of appropriate higher layers. In spite of not requiring significant changes in network elements, overlay mechanisms add complexity to the system and are not scalable. Such overlay deployments add excessive signaling load, energy consumption and CPU/memory overhead with control functions, most of them in a centralized way [1]. Moreover, the heterogeneity of current scenarios require cross-layering, where information can be potentially exchanged by non adjacent layers [2]. Session quality can then be adapted based on parameters from different layers: session (codec, destination, policy); network (bandwidth, delay, jitter and loss); data-link (SNR, frame error, priority queuing, burst size); and physical (velocity, location, humidity). However, current "overlay architecture" concepts make cross-layering design evaluation an outstanding challenge [3].

Such limitations of current Internet motivated multiple proposals for a clean-slate architecture embedding major networking innovations for the efficient deployment of future scenarios [4]. Within the FP7 4WARD project [5], a new connectivity paradigm called Generic Path (GP) is proposed, which aims to optimize connectivity by deploying a unique way to provide communication. Data transport over GPs is possible by mapping sessions into physical resources, such as links, network interfaces, routers, etc. Thus, GP expects to drastically facilitate the specification and inclusion of new applications and services in an heterogeneous environment.

The success of the GP architecture depends on the provisioning of efficient mechanisms for mapping physical resources to comply to each session requirements, in order to deliver end-to-end multi-party data transport with guaranteed QoS over the time. This paper proposes QoS Routing and Resource Control (QoS-RRC) for allowing the dynamic setup of GP sessions in terms of QoS and multi-party connectivity over heterogeneous environments. QoS-RRC takes distributed decisions based on a new over-provisioning specification of QoS control and transport approaches, solving scalability issues, and is supported by routing strategies with robust, reliable and scalable functionalities. Thus, QoS-RRC is expected to allow the support of many types of time-sensitive sessions while drastically reducing signaling load throughout the network and optimizing state and CPU overheads. Initial QoS-RRC performance evaluation using NS-2 [6] demonstrated performance benefits in terms of overall signaling and state overhead reduction in comparison with a value-added per-flow approach.

This paper does a brief description of the Generic Path architecture, describing its mechanisms and elements. Our main proposal, QoS-RRC is then presented, and its 
evaluations results on NS-2 are presented. Final remarks and future work conclude this text.

\section{GENERIC PATH OVERVIEW}

The Generic Path architecture aims for the support of the growing diversity of applications and network technologies, and their subsequent interaction. A Generic Path is an object abstracting a communication path [7]. It is a combination of both transportation - generalizing transport mediums (e.g. physical wires, wireless channels) as virtual connections and transformation - mapping the corresponding capabilities (e.g. encapsulation, encryption, aggregation) - functions.

These and other characteristics of the GP architecture make it a very powerful one for network design, selectively hiding management complexity as desired. The entities that allow this and their functions will now be described.

\section{A. GP Architecture and its elements}

In the GP architecture, nodes (terminal or network ones) are represented by Node Compartments (Node CT). Entities (ET), which model a running service at any level and generalize data processing functions for communication, exist within such Node CTs and communicate with other ETs, either horizontally - by GPs - or vertically - interservice or inter-technology communication using Hooks. ET functions include name resolution, routing and addressing, and they access GPs through End Points (EPs.) A GP is an abstraction of the service path travelled by data, and generalizes a data transport and/or a transformation service across a network, by comprehending the set of necessary resources that provide end-to-end communication between two or more client ETs.

A GP may be composed by other GPs, each implementing a different service in a different technology, that way allowing a complete view of the data path (see Figure 1). Two or more GPs may be connected using a Mediation Point (MP) for pipelining data between them - GP multiplexing. GPs store their characteristics (such as composing GPs, EPs and resource attributes, such as QoS parameters or connection capabilities) in GP Managements Records (GPMR). These records are organized and located referring to the Master Record (MR), which holds a nodewide view of the GPs and its resources [8].

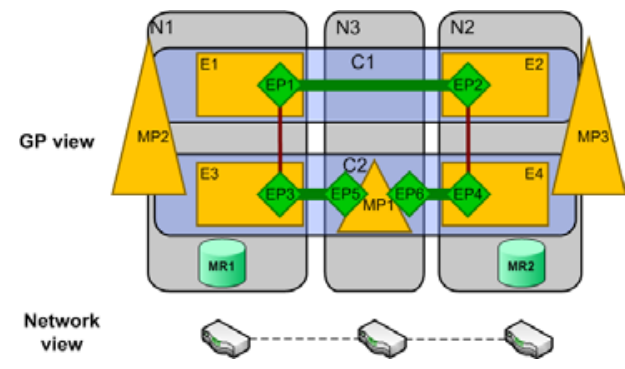

Figure 1. GP Network Example Scheme

\section{B. GP Resource Management Database}

At the highest abstraction level, a GP is the result of an aggregation of lower level GPs, one for each of the technologies that the communication depends on (TCP, Ethernet, Optical Fiber, etc). The managed resources range from metrics such as throughput, SNR and end-to-end delay, to policy data, such as packet priority level, privacy and identifying keys.

On the other hand, the traffic flow is ensured by the creation and management (modification, destruction) of multiple GPs, and for this a framework for collecting and controlling the diverse path information is logically needed. MR [8] is a database at each node holding the relationships between each GP's resources. This structure holds a view of all GPs composing the same E2E communication by holding a list of the GP management records (GPMRs), one for each sub-GP. By adding the notion of Compartment Record (CTR), a record listing the GPMRs running in the same CT, it is possible to establish a distributed control scheme. This way, a MR points to a list of CTRs, one for each CT in which the Node CT participates. Such framework brings an innovative approach to current networks, providing a unified control framework for the communication path. Besides, each GP needs proper control, and as the element that stores all relevant information, the GPMR can provide such a feature.

The next section presents the focus of this paper, the routing-enabled QoS control proposal for processing network resources as support for efficient GP.

\section{QOS-RRC DESCRIPTION}

QoS-RRC aims to enhance the GP architecture with capabilities for providing communications fulfilling the requirements of interested entities by means of efficient network resources provisioning. For this, QoS-RRC implements a routing-enabled QoS control approach following a dynamic and scalable over provisioning-centric strategy [9][10] to handle bandwidth and path allocation. For bandwidth provisioning, a dynamic per-class overreservation is deployed and admission control allows setting up multiple sessions without per-flow signaling. By bootstrapping a routing table with shortest QoS-aware communication paths inside the network, data paths provisioning is possible. Therefore, reliable routing can be done more efficiently by means of a broadly network view of current QoS capabilities, in contrast to current solutions, which only keep next hop information, with resource capabilities being classified by inefficient metrics and maintained by constant flooding operations. Fig. 2 shows the architecture of QoS-RRC compliant with the GP framework.

QoS-RRC is hosted in nodes implementing MPs, and allows routing and QoS control as in the following. Dynamic routing events can be provided by asynchronous signaling, such as when a network interface goes down or up. Moreover, periodic signaling is used to collect QoS capabilities and routing information. Finally, QoS enforcement to install or modify resources is done ondemand. As the 4WARD project envisions totally optical 
core network elements, decisions are taken in network borders. Thus, QoS-RRC agents are hosted in ingress and egress routers, and the core implements lightweight agents addressing message receptions for resource enforcement, as well as generating asynchronous alarms.

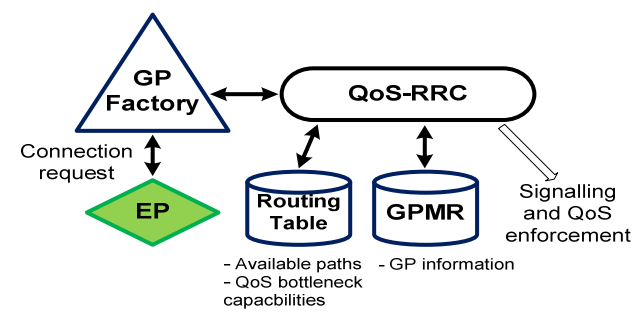

Figure 2. QoS-RRC architecture

\section{A. QoS-aware Routing}

The main idea of the routing-enabled QoS control mechanism of QoS-RRC consists in bootstrapping a routing table with shortest QoS-aware path information, including the IP address of each router along the path and correspondent bottleneck's QoS capabilities (per-class available bandwidth and rates of delay, jitter and loss). For such, each ingress node floods the network, and each visited node initializes per-class over-reservations (taking into account an assigned factor, such as $1 / 2$ or $1 / 4$ of the local link capacity), adds its IP and current QoS conditions to the message, and forwards it downstream. After the operations described above succeed, the egress node signals the ingress one. In order to avoid infinite looping and redundant information storage in flooding, each node must only inspect messages once and a limited number of hops (as in TTL) can be assigned to avoid long paths. This flooding cycle provides information about unicast paths, composed by one ingress router, a set of core routers and one egress router. After finishing the flooding cycle, each ingress router implements an algorithm that takes unicast paths to generate all possible QoS-aware multicast trees (matching it and available egress nodes). QoS-RRC filters the information, keeping only best multicast trees for avoiding excessive matching's. For instance, trees with ingress routers in the middle are discarded for keeping only downstream transport. Afterwards, QoS-RRC signals each path to setup multicast state accordingly.

A connectivity control mechanism is supported by QoSRRC, allowing the aggregation of multiple GPs in the same tree. This aggregation technique aims to reduce state and processing overhead inside the network (as of legacy IP multicast), thus allowing scalable multi-party support. Such mechanism also improves resilience, as sessions can berouted seamlessly under network dynamic events. For instance, a GP can be allocated into another path due to link failure of previous path, and a session can request the allocation of the GP to the original path when the link comes up again (such decision is out of QoS-RRC scope). Thus, QoS-RRC allows fast resilience support and intermittent communications.

\section{B. Over Provisioning-centric QoS Control}

After all over-reservations and routing table completion, ingress routers remain waiting for session setup requests in order to start control operations. The QoS-RRC controls QoS by associating admission control and dynamic per-class bandwidth over-reservation resizing, allowing QoS-aware routing for session propagation over appropriated GPs, while keeping overall system performance. The over-reservations are resized whenever a class cannot admit a GP request. QoS-RRC resizes a class over-reservation by taking into account statistics about network conditions, resource demands and class-policies. The integration of admission control and per-class over-reservation foresees network performance improvements, where multiple GPs can be installed without per-flow signaling, as much as possible.

During a GP creation, the GP Factory (the entity setting the GP) triggers QoS-RRC with a GP request carrying information about source and destination IP, as well as QoS requirements (bit-rate, class of service, maximum delay, jitter and packet loss rate). After that, QoS-RRC chooses the most appropriate path among all the available ones in the routing table, accepts the request and then triggers the Mediation Point (MP) to establish the connection (setup the tunneling for aggregation). After setting all configurations, the GP factory is fed back to map the referred GP into the path provided by QoS-RRC, to further allow session propagation accordingly. In case of failing the admission control in all candidate paths, QoS-RRC attempts to adapt resources of the path by resizing the over-reservation accordingly. In case of no success, the session is denied.

\section{PERformance EVAluation}

The performance evaluation of QoS-RRC was carried out in Network Simulator v2 (NS-2) with GP and QoS-RRC extensions. The simulation model uses a random topology of 14 routers with links of $100 \mathrm{Mb} / \mathrm{s}$ and varying propagation delay (generated by BRITE), scaled with 1,000 UDP multicast sessions with $256 \mathrm{Kbps}$ of constant bit-rate for different classes (following a Poison distribution). For classbased traffic differentiation, DiffServ with WFQ scheduling discipline is used with the following class configurations: one EF-alike (Premium); two AF-alike (Gold and Silver); and one best-effort. In order to avoid class starvation, each class was assigned with a maximum reservation threshold of $20 \%$ of the link capacity, thus leaving best-effort with $40 \%$ of the resources ensured. Three sets of analysis have been deployed to study the impact of QoS-RRC in the simulation model, and results have been collected taking a confident interval of $95 \%$.

\section{A. Analysis of the Results}

The signaling load is an important measure to study the impact of QoS-RRC in the overall system performance, since processing burden overloads with the increasing amount of signaling. The methodology applied in this set of experiments is to compare QoS-RRC signaling behavior against a relevant per-flow solution. In this sense, Multi- 
service Resource Allocation (MIRA) [11] was chosen due to its improvements against the state of the art (e.g., RSVP [12]). Fig. 3 shows the analysis in signaling load.

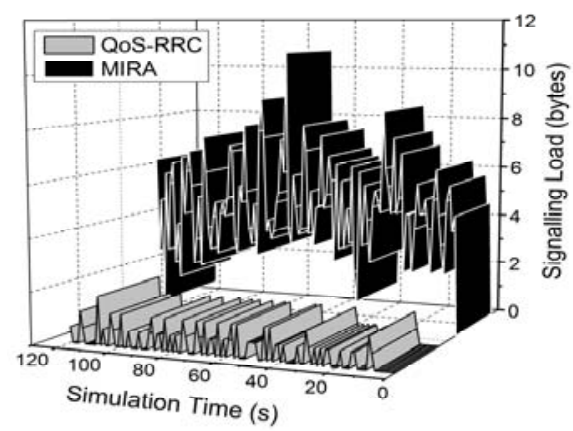

Figure 3. Signaling load of MIRA and QoS-RRC

The numerical results show that the signaling load of MIRA and QoS-RRC in the simulation average per second 4.72 Bytes and 0.82 Bytes respectively. Thus, the over provisioning-centric QoS control strategy of QoS-RRC reduces in more than 5 times the amount of signaling exchanges. Such reduction is achieved through bandwidth over-provisioning, which allows admitting multiple sessions without any signaling. In contrast, the per-flow basis of MIRA introduced much more signaling. Thus we can conclude that QoS-RRC drastically improve overall network performance over per-flow operations.

Fig. 4 depicts bandwidth consumption analysis in the simulation.

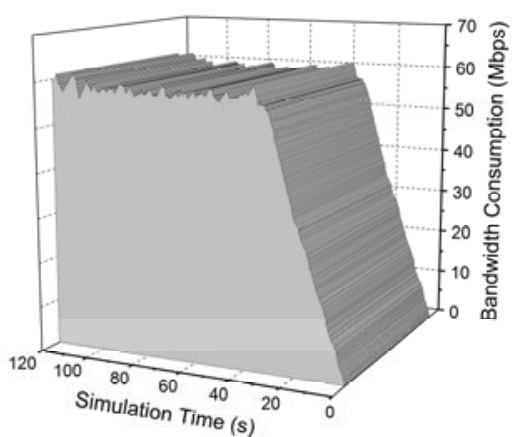

Figure 4. QoS-RRC Bandwidth Consumption in the Simulation Model

The bandwidth consumption is used as a measure to testify the influence of QoS-RRC in resource allocations for data delivery. Fig 4 exhibits that the QoS-RRC admission control prevented congestions by not allowing excessive traffic of sessions, never exceeding 60Mbps. The numerical results in simulation attested that even under congestion periods of some classes (not shown in Fig. 6), QoS-RRC managed residual bandwidth to admit sessions (66\% of all 1,000 GP requests). Therefore, we can conclude that QoSRRC allows better bandwidth utilization than per flow analysis by avoiding waste of resources even during congestion periods or presence of residual bandwidth.

\section{CONCLUSION AND FUTURE WORK}

The European FP7 project 4WARD is specifying novel network architectures for replacing the current Internet model. One of its greatest advances refers to the communication mechanism called Generic Path (GP). The success of GP architecture depends upon the specification of routing and QoS control mechanisms, in order to correctly map physical resources (links, interfaces, etc.) for supporting the expected types of sessions. This paper presented the Routing and dynamic Resource Control (QoS-RRC), a suite of mechanisms addressing routing-enabled QoS control approach to fulfill the above requirements. QoS-RRC integrates QoS routing and resource over-provisioning to allow efficiently GP mapping into physical resources. QoSRRC was evaluated in NS-2, and demonstrated the accomplishment of its expected benefits in terms of signaling load and bandwidth allocation control.

As future work, the QoS-RRC performance evaluation points to prototyping analysis in order to study functionalities with more accuracy.

\section{ACKNOWLEDGMENT}

This work is supported by the FP7 4WARD project and FCT POPH/FSE - QREN - Tipologia 4.1.

\section{REFERENCES}

[1] Manner, J., and Fu, X. (2005), "Analysis of Existing Quality-ofService Signaling Protocols”, IETF RFC 4094, May 2005

[2] Kawadia, V. and Kumar, P.R , "A Cautionary Perspective on CrossLayer Design” IEEE Wireless Communications., vol. 12, no. 1, Feb. 2005, pp. 3-11

[3] Foukalas, F.; Gazis, V.; Alonistioti, N "Cross-layer design proposals for wireless mobile networks: a survey and taxonomy", Communications Surveys \& Tutorials, IEEE Volume: 10 , Issue: 1

[4] Roberts, J., "The clean-slate approach to future Internet design: a survey of research initiatives”, Annals of Telecommunications, 2009 - Springer.

[5] The FP7 4WARD Project, http://www.4ward-project.eu/

[6] The NS-2 Home Page, http://www.isi.edu/nsnam/ns/

[7] Randriamasy, S. et al., Mechanisms for Generic Paths, 4WARD deliverable D-5.2, December 2009

[8] Figueiredo, S., Lourenço, J., Aguiar, R. L., and Neto, A., "Taxonomy for GP-aware mobility". Proc. of the First International ICST Conference on Mobile Networks and Management, 2009

[9] Neto, A., Cerqueira, E., Monteiro, E., Mendes, P., (2008), "Scalable Resource Provisioning for Multi-user Communications in Next Generation Networks”, In: IEEE Globecom 2008, New Orleans, LA, USA

[10] Neto, A., Cerqueira, E., A., Monteiro, E., Mendes, P., (2008), "Scalable Multimedia Group Communications through the Overprovisioning of Network Resources”, In: 11th IFIP/IEEE MMNS 2008, 2008, Samos Island

[11] Neto, A., Cerqueira, E., Rissato, A., Monteiro, E., Mendes, P., (2007), "A Resource Reservation Protocol Supporting QoS-aware Multicast Trees for Next Generation Networks" In: 12th IEEE ISCC, 2007, Aveiro, Portugal

[12] Brades, R., Zhang, L., Berson, S., Herzog, S., Jamin, S. (1997) "Resource ReSerVation Protocol (RSVP) -- Version 1 Functional Specification”, IETF RFC 4094, September 1997 